\title{
Turbulent Heat Transfer of Supercritical Fluids: Fundamentals and Modeling
}

\author{
Bofeng Bai \\ State Key Laboratory of Multiphase Flow in Power Engineering \\ Xi'an Jiaotong University \\ Xi'an, Shaanxi, China \\ bfbai@mail.xjtu.edu.cn
}

Supercritical fluids have become promising working mediums in many industrial fields such as the coal-fired power generation, nuclear power system, hydrogen production and distributed energy. The accurate estimation of the flow and heat transfer characteristics of supercritical fluids is necessary for the design and optimization of the systems and devices.

At supercritical state, a transition from a liquid-like to a vapor-like substance occurs continuously with the increasing temperature. And during this transition, all the thermo-physical properties of the fluids vary significantly. The region where this significant change of the thermo-physical properties happens is called "the pseudo-critical region". The existed turbulent models (including RANS Model, Reynolds Stress Model and Algebraic Flux Model) could not give satisfying results for the turbulent heat transfer at the pseudo-critical region.

In the first part of this lecture, we will show the special turbulence fundamentals of supercritical fluids: i) The density fluctuation is significant so that the Morkovin's hypothesis is no longer validated, as a result the dynamics, transport and structures characteristics of the turbulence have essential differences with those of the conventional compressible turbulence. ii) The strong body force (buoyancy force) caused by the density variations systemically influences the transport of the turbulence. Namely, the body force could influence the motions of the eddies structures, this increases the turbulent kinetic energy and Reynolds heat flux transports induced by the pressure fluctuation; The obvious countergradient transports of the turbulent kinetic energy and scalar energy could be observed under the stratification effect. In addition, the turbulent motions are anisotropy on the small scales, which does not match with the Kolmogorov's first hypothesis.

Based on the turbulence fundamentals of the supercritical fluids, the deficiencies of the hypothesis in the existed turbulent models are analysed: i) Morkovin's hypothesis in RANS model, RSM and ASM. ii) Gradient diffusion hypothesis in RANS model, RSM and AFM. iii) Energy cascade hypothesis in RANS model. iv) Local equilibrium hypothesis in AFM. v) Isotropy hypothesis in RANS model, RSM and AFM. Finally, we try to introduce the accurate turbulence model. Some new research progress about the modifications of the turbulent models by our team and other researchers will be presented in this lecture. 